\title{
Abstracts of the 20th Nippon Medical School Foundation Academic Meeting for Foreign Researchers
}

Date: 23rd January, 2010

Hours: 10 : 30 A.M.-5 : 05 P.M.

Place: Nippon Medical School Lecture Room 3

Management: Foreign Researchers Belong to Nippon Medical School Foundation

\section{Epidemiological Characterization of Campylobacter spp. Isolated from Retail Chicken Cuts in Western Tokyo}

Bongkot Noppon $^{1,2}$, Yasushi Kataoka ${ }^{1}$ and

Takuo Sawada ${ }^{1}$

${ }^{1}$ Division of Veterinary Microbiology,

Nippon Veterinary and Life Science University

${ }^{2}$ Division of Veterinary Public Health,

Faculty of Veterinary Medicine,

Khon Kaen University

Objective: The aim of this study was to clarify the epidemiological characteristics and antimicrobial susceptibility of Campylobacter spp. isolated from chicken cuts obtained in western Tokyo.

Materials and Methods: A total of 260 chicken cut samples were collected from 20 retail stores in western Tokyo on 3 days in October and December, 2006, and Campylobacter spp. were isolated with selective media. The bacterial species were identified with PCR. The PCR-RFLP fingerprint of flaA was used for clustering. Penner's method was employed for serotyping, and antimicrobial susceptibility was determined with the agar dilution method.

Results: Of the samples, 168 (65\%) were positive for Campylobacter species. Of 129 isolates from the positive samples, 121 (94\%) were C. jejuni and 8 (6\%) were $C$. coli. Sixty-eight isolates of $C$. jejuni were subjected to further characterization; serotype B was the most common (30\%), and serotype $\mathrm{Y}$ was the second-most common (10\%). The fingerprint divided the isolates into 2 major clusters, I (29\%) and II (71\%). An antimicrobial susceptibility test revealed that $33 \%, 48 \%$, and $1 \%$ of the isolates were resistant to ofloxacin, doxycycline, and erythromycin, respectively, and that none was resistant to chloramphenicol. Single antimicrobial resistance was the most evident feature (33\%), and only $1 \%$ of the isolates had a co-resistant profile to 3 antimicrobials.

Conclusions: C. jejuni was the most common Campylobacter species isolated from the chicken cut samples investigated in the present study. The major serotype of the isolates was $\mathrm{B}$, and most of them belonged to cluster II of flaA. A particularly high rate of isolates was found in the samples from 1 specific store. There were no relationships among the serotype, flaA cluster, and antimicrobial susceptibility of the C. jejuni isolates.

2. Comparative Studies of Canine Mesenchymal Stem Cells Derived from Bone Marrow and Adipose Tissue

\author{
Dongwei Zhao, Yasuji Harada, \\ Yasushi Hara, Takuya Yogo, \\ Yoshinori Nezu and Masahiro Tagawa \\ Division of Veterinary Surgery, \\ Nippon Veterinary and Life Science University
}

Mesenchymal stem cells (MSCs) can be harvested from various mesenchymal tissues, such as bone marrow and adipose tissue. We hypothesized that MSCs harvested from different organs show different characters and abilities. In this study, we used bone marrow-derived mesenchymal stem cells (BMSCs) and adipose-derived mesenchymal stem cells (AMSCs) from beagles. Comparative measurements were made of cell DNA content (proliferation) and ALP activity (osteogenesis) in BMSCs and AMSCs. The cells were analyzed by flow cytometry, and then plated at $5 \times 10^{3}$ cells $/ \mathrm{cm}^{2}$ in multi-well plates for osteogenetic culture in vitro. No character differences between BMSCs and AMSCs were identified on flow cytometry analysis. AMSCs showed a higher proliferation ratio but lower ALP activity than BMSCs.

Journal Website (http://www.nms.ac.jp/jnms/) 


\section{Changes in Ultrasonic Vocalizations Emitted by Rats during the Pre-weaning Period}

\section{Pudcharaporn Kromkhun ${ }^{1,2}$, Masahiro Katou', Makoto Yokosuka ${ }^{1}$, Tomoaki Nakada ${ }^{1}$ and Toru Saito ${ }^{1}$ \\ ${ }^{1}$ Division of Comparative and Behavioral Medicine, Nippon Veterinary and Life Science University \\ ${ }^{2}$ Division of Physiology, Faculty of Veterinary Medicine, \\ Kasetsart University}

Rodent pups emit ultrasonic vocalizations (USVs) in such situations as separation from the nest and cold stress. These USVs have been reported to be an essential form of communication between the pups and their mothers. In this study, we attempted to measure ontogenetic changes in USVs emitted by infant Wistar-Imamichi rats isolated from their mothers during the pre-weaning period. The number of calls, the median pitch frequency, and the lowest pitch frequency peak were measured on days $1,3,5,7,10,12$ and 14 of age. Each pup was place in a cold beaker, and USV measurements were recorded for $5 \mathrm{~min}$. The pups' body weight increased significantly with age, except on days 710. The number of calls increased to a peak on day 5 , and then decreased gradually. The median frequency of the calls decreased gradually over the first 12 days, and then showed a small increase on day 14 . The lowest frequency peak shifted gradually to lower levels from day 1 through day 12 , and then shifted slightly higher on day 14 , with an overall trend toward median frequency with aging. The present study is the first to clearly establish that ontogenetic changes take place in USVs emitted by infant rats.

\section{Study of Genetic Diversity in Mongolian Native White Horses: Polymorphism of Coat-color Genes (Extension, Agouti and Brown)}

Siqingaowa ${ }^{1}$, Harutaka Mukoyama ${ }^{1}$ Manglai ${ }^{2}$, Junji Ueda ${ }^{3}$ and Toru Saito
${ }^{1}$ Division of Comparative and Behavioral Medicine,

Nippon Veterinary and Life Science University

${ }^{2}$ Inner Mongolia Agricultural University

${ }^{3}$ Rakuno Gakuen University

Many Mongolian native horses with various coat colors inhabit the grasslands in Inner Mongolia. Among these semi-wild horses, about 100 head of white-coat horses live as a herd in WestWuzhumuqin. It is known that the white coat is the result of several gene mutations that inhibit the development of melanocytes containing pigment granules. In this study, the 3 basic coat-color genes (Extension [MC1R], Agouti [ASIP], and Brown [TYRP1]) were analyzed to determine their frequency and polymorphisms in Mongolian native white horses. Peripheral blood was collected from 50 horses, and DNA was isolated from the blood by phenol-chloroform extraction. PCR amplifications of MC1R, ASIP and TYRP1 genes were performed with specific primers, and nucleotide sequencing of the PCR products was carried out with a BigDye terminator cycle sequencing kit. Genotyping of the 3 coat-color genes was performed by the PCR-RFLP and PCR-AFLP methods. Gene frequency and the Hardy-Weinberg equilibrium were calculated by statistical analysis.

The horses were classified into 3 groups: dominant white horses (4\%); grey horses (88\%); and sabino horses (8\%). A single nucleotide polymorphism (SNP) (C901T) was found in the second domain region of the MClR gene and was classified into $\mathrm{EE}, \mathrm{Ee}$ and ee phenotypes. The gene frequency of $E$-allele was 0.57. A deletion of $11 \mathrm{bp}$ was detected in exon 2 of the ASIP gene. The gene frequency of $A$-allele was 0.87, and a deviation in the Hardy-Weinberg equilibrium was found between the observed value and the expected value of the ASIP genotypes. An SNP (C189T) was also found in exon 2 of the TYRP1 gene and was classified into $\mathrm{BB}$ and $\mathrm{Bb}$ phenotypes. No bb phenotype was found. The gene frequency of $B$-allele was 0.98 . We speculate that the Mongolian native white horse has the major coat-color genes, but that their expression is inhibited by the white coat color-related genes. 


\section{The Essentials for Delivering High-quality Tertiary Education: An Analysis of the Nippon Medical School Curriculum}

\author{
Zheng Shi, Fanlai Cui, \\ Yi Ye and Yokichi Ohno \\ Department of Legal Medicine, \\ Nippon Medical School
}

Higher education should be adapted to the requirements of society and the development of a nation's youth. Therefore, the cornerstone of higher education is not only delivering knowledge to students, but also educating them. In order to remain competitive in a society facing declining numbers of young people, the most important issue for institutions of higher education is to have definite goals, complete teaching segments, and outstanding features and characteristics. Nippon Medical School's reforms are based on the goal of "nurturing medical professionals with experience and excellent skills." After 10 months' experience of studying at Nippon Medical School, the author is greatly impressed by the $\mathrm{T} /$ Each Other Program, which breaks away from the confines of grades and campus. As an outstanding feature of Nippon Medical School, this program provides wide-ranging education at real medical care facilities. It has greatly enhanced students' abilities to think for themselves, learn for themselves, and solve problems. It has also improved their clinical performance, and communication skills with patients.

6. Effect of Injecting an Orexin-saporin Infusion into the Medial Preoptic Area and the Bed Nucleus of the Stria Terminalis on Male Rat Sexual Behavior

Dilip Rai, Yuriko Kato, Yasuhiko Kondo and Yasuo Sakuma

Department of Physiology,

Nippon Medical School

Orexin is a neuropeptide involved in sleep-wake regulation and feeding behavior. Neurons expressing orexin are exclusively located in the lateral hypothalamus and have dense and reciprocal projections to the preoptic and other hypothalamic areas. Recent studies have also demonstrated the role of the orexinergic system in the reward system. In our current study, we examined the effect of injecting orexin-saporin conjugate, a targeted toxin for orexin-receptor positive neurons, into the preoptic area (POA) and the bed nucleus of the stria terminalis (BNST) on male rat sexual behavior. While injection into the POA severely suppressed overall male sexual behavior, injection into the BNST specifically prolonged latency time for ejaculation without any other disturbances. Our data suggest that orexin exerts a facilitatory influence on the preoptic neural substrates essential for male sexual behavior as well as on the hypothalamic ejaculatory circuits.

\section{Systemic Cancer Gene Therapy for Lymphoma Using AAV Vectors Expressing mda-7/IL24}

Nanya Wang, Koichi Miyake and

Takashi Shimada

Department of Biochemistry and Molecular Biology,

Nippon Medical School

Melanoma differentiation-associated gene-7 / interleukin-24 (mda-7/IL24) selectively induces apoptosis in cancer cells without harming normal cells. It also exerts immunomodulatory and antiangiogenic effects, as well as potent antitumor bystander effects, making it an ideal candidate for use in a new anticancer gene therapy. To examine the feasibility of using adeno-associated virus (AAV) vectors expressing mda-7/IL24 in systemic cancer gene therapy for lymphoma, we generated an AAV type 8 vector expressing mda-7/IL24. First, we evaluated the efficacy of this vector by treating lymphoma cells with a medium conditioned with AAV-8-mda7-transduced C2C12 cells. TUNEL assay confirmed apoptosis of the lymphoma cells. We also established a murine model of lymphoma by injecting luciferase-expressing A20 tumor cells (murine lymphoma cell line: A20/Luc) into the caudal vein of BALB/C mice. After administering a single injection of AAV vector into the quadriceps muscle, we analyzed tumor growth with an IVIS system and measured IL-24 serum levels by ELISA. We are now 
analyzing the suppression of tumor growth and survival effect in this lymphoma model.

8. The Influence of Stearoyl-CoA Desaturase Genotype and Gene mRNA Expression on M. longissimus thoracis Fatty Acid Composition in Holstein Steers

Ju-hwan Lee, Jin-suk Jeong,

Ichiro Yamamoto, Toshihiro Nade and

Nobuhiro Kimura

Division of Animal Nutrition,

Nippon Veterinary and Life Science University

Purpose: Recently, it has been suggested that the genotype and mRNA expression of stearoyl-CoA desaturase (SCD) genes significantly affects the level of adipose tissue monounsaturase fatty acid (MUFA) in Japanese Black cattle. However, the significance of these factors has not yet been investigated in relation to Holstein steers, which play a critical role in the Japanese beef market: approximately $40 \%$ of the beef consumed in Japan is from Holsteins. In this study, we investigated the influence of SCD genotype and mRNA expression on the fatty acid composition of $M$. longissimus thoracis in Holstein steers.

Methods: The average age and live weight of the animals were 10.3 months and $476.0 \mathrm{~kg}$, respectively. To determine SCD genotype, we applied PCR-RFLP methods to genomic DNA isolated from blood samples from Holstein steers $(n=15)$. qRT-PCR analysis was performed with cDNA synthesized from intramuscular adipose tissue collected via biopsy between the $12^{\text {th }}$ and $13^{\text {th }}$ thoracic vertebrae $(n=6)$. Gas chromatography was used to calculate the fatty acid composition by the area normalization method.

Results: With regard to SCD genotypes, the allele frequencies of AA type and AV type were $46 \%$ and $53 \%$, respectively; VV type was not detected. No differences in MUFA composition between the genotypes were noted at 10 months of age, but at 14 months AA type was more common than AV type $(\mathrm{P}<0.05)$. This study suggests that SCD genotype and age affect the fatty acid composition of M. longissimus thoracis in Holstein steers.
9. Prenatal Dexamethasone Exposure Affects Expression Levels of Brain-derived Neurotrophic Factor and Neurotrophin-3 in the Hippocampus and Medial Prefrontal Cortex in Rats

Mingyan Liu, Masatoshi Nagano and Hidenori Suzuki

Department of Pharmacology, Nipppon Medical School

It has been suggested that prenatal stress affects the development of the central nervous system (CNS). We recently reported that prenatal dexamethasone (DEX) exposure, which mimics prenatal stress, induced anxiety-like behaviors in male rat offspring at 10 weeks or later after birth. Since neurotrophic factors (NTFs) play important roles in the development and plasticity of the CNS, we hypothesized that NTFs might be involved in the pathogenesis of DEX-induced anxiety behavior. To test this hypothesis, we examined the expression levels of brain-derived neurotrophic factor (BDNF) and neurotpphin-3 (NT-3) in the hippocampus and medial prefrontal cortex (mPFC) of male and female rat offspring at 4 weeks after birth. Prenatal DEX exposure decreased BDNF in the hippocampus and $\mathrm{mPFC}$ in both sexes. On the other hand, decreases in NT-3 levels were detected in the male mPFC and the female hippocampus. Collectively, prenatal stress induced with DEX may cause anxiety behavior in adulthood in association with a precedent change in NTF expression during development.

10. Food Intake, Serum Leptin Levels, and Leptin mRNA Expression during the Sexual Cycle in Female Rats

Wirasak Fungfuang ${ }^{1}$, Tomoaki Nakada ${ }^{1}$, Pudcharaporn Kromkhun ${ }^{1,4}$, Misao Terada ${ }^{2}$, Richard S. Nakamura', Kaoruko Hashimoto', Fujiro Sakurai ${ }^{3}$, Makoto Shimizu', Nobuhiro Nakao ${ }^{5}$, Makoto Yokosuka ${ }^{1}$ and Toru Saito ${ }^{1}$ 
${ }^{\mathrm{l}}$ Division of Comparative and Behaviral Medicine,

Nippon Veterinary and Life Science University

${ }^{2}$ Division of Laboratory Animal Science,

Nippon Medical School

${ }^{3}$ Division of Animal Science

Teikyo University of Science and Technology

${ }^{4}$ Division of Physiology,

Kasetsart University

${ }^{5}$ Division of Animal Physiology,

Nippon Veterinary and Life Science University

The aim of this study was to investigate food intake, serum leptin levels, and leptin mRNA expression during the sexual cycle in female WistarImamichi rats aged 8-10 weeks. Food intake was measured in both light and dark phases (lights on at $07: 00$ and off at $19: 00)$ through the 4-day estrous cycle of the rats. We measured serum leptin levels with ELISA and leptin mRNA expression with realtime PCR in the diestrous and proestrous stages. Food intake was significantly greater in the dark phase than in the light phase during the sexual cycle, and the food intake of proestrous females was significantly lower in both the light and dark phases than that of the other groups. Serum leptin concentrations in both phases were significantly higher in the proestrous than in the diestrous groups. There was a significant increase in leptin mRNA expression in adipose tissue during the proestrous period compared with the diestrous period. These findings suggest that increased leptin mRNA expression and serum leptin levels induced by estrogen in the proestrous stage may play a role in the regulation of appetitive behavior.

\section{Phylogenetic Analysis of the actA Region of Listeria monocytogenes}

\section{Otgonchimeg Batmunkh, Keisuke Fujita, Takashi Takano, Mariko Mochizuki, Yoshitsugu Ochiai, Ryo Hondo and Fukiko Ueda Division of Veterinary Public Health, Nippon Veterinary and Life Science University}

Introduction: The epidemiology of food-borne diseases caused by Listeria monocytogenes (Lm) has been based on the evolutionary classification of Lm into 3 large groups. The aim of this study was to clarify the classification of Japanese Lm strains (JPN strains). At the $18^{\text {th }}$ and $19^{\text {th }}$ Nippon Medical School Medical Research Conference for Foreign Researchers, we reported our classification of $\mathrm{Lm}$ using the iap and sigB genes. In the present study, we attempted to classify Lm using the actA gene.

Materials and Methods: JPN strains have been classified into 34 genotypes using the iap gene, and we selected a representative strain from each genotype. A nucleotide sequence of $561 \mathrm{bp}$ within the act $\mathrm{A}$ region was determined using extracted genomic DNA. The sequences of USA strains were obtained from www.pathogentracker.net. Phylogenetic analyses were performed based on the sequences.

Results and Discussion: 1) Since the iap-C-group (4 strains) in JPN strains is not amplified by USA primers, the genomic structure of this group appears to be different from that of USA strains. 2) The USA strains were classified into 4 groups. Four of 29 JPN strains were shown to be different from USA strains in that they formed 3 and 1 new clusters (in "a" and "b" groups, respectively). Consequently, in this study we showed that the genotypes of some JPN strains differed from those of USA strains.

12. Phialophora verrucosa Isolated from Cultured Japanese Flounder (Paralichthys olivaceus) in Ehime Prefecture, Japan

Nguyen Truc Phuong ${ }^{1,2}$, Osamu Kurata ${ }^{1}$ Shinpei Wada', Ayako Sano ${ }^{3}$ and Kishio Hatai ${ }^{1}$

${ }^{1}$ Division of Fish Diseases, Nippon Veterinary and Life Science University ${ }^{2}$ College of Aquaculture and Fisheries, Cantho University

${ }^{3}$ Research Center for Pathogenic Fungi and Microbial Toxocoses, Chiba University

We examined Japanese flounder (Paralichthys olivaceus) cultured in a net cage in Ehime Prefecture, Japan after receiving a report of small non-fatal jaw lesions in some of them in March, 2009. We found fungal hyphae in the lesions under a microscope. 
The strain NJM 0951 isolated from the inside of the jaw formed a funnel-shaped collarette, and was identified as Phialophora verrucosa from its morphological characteristics. Additionally, rRNA genes from the ITS and D1/D2 regions were analyzed according to a routine method. The isolate was perfectly consistent with $P$. verrucosa. The fungus grew well at $25^{\circ} \mathrm{C}$ to $30^{\circ} \mathrm{C}$ but only weakly at $10^{\circ} \mathrm{C}$ and $37^{\circ} \mathrm{C}$ on potato dextrose agar (PDA). Growth was faster on PDA than on peptone yeast extract glucose seawater agar. The fungus grew well on PDA agar with $1 \%$ to $2 \% \mathrm{NaCl}$, but weakly on PDA with $0 \%$ and $5 \% \mathrm{NaCl}$. To our knowledge, this is the first report of $P$. verrucosa infection in fishes.

\section{Development of a Novel Mutation Detection System in the Vascular Type of Ehlers-Danlos Syndrome (vEDS, EDS Type IV) Using High- resolution Melting Curve Analysis}

\section{Banyar Than Naing, Atsushi Watanabe and}

Takashi Shimada

Department of Biochemistry and Molecular Biology, Nippon Medical School

The vascular type of Ehlers-Danlos syndrome (vEDS, EDS type IV), which is caused by a heterozygous mutation of type 3 procollagen (COL3A1), is an autosomal dominant genetic disorder that causes blood vessel rupture, intestinal rupture, and uterine rupture during pregnancy, all of which can lead to sudden death. Two thirds of COL3A1 mutations are missense mutations of glycine, present in every 3-amino acid in the triple helix region, to other amino acids; the remaining $1 / 3$ are splice site mutations in the exon-intron junction. Up to now, most analyses of COL3A1 mutations have relied on total RNA extracted from patients' fibroblast cells. Recently, a mutation scanning system called highresolution melting curve analysis (hrMCA) has been developed. This system uses PCR- and DNAintercalating fluorescence released during doublestranded DNA melting, which creates different melting curve patterns depending on nucleotide differences between a control subject and the patient. We have carried out mutation screening for
COL3A1 with the hrMCA method using genomic DNA. First, we designed primer pairs for 52 exons of the whole COL3A1 gene, covering all exon-intron junctions. PCR was performed with a DNAintercalating fluorescent dye (LCGreen), and a light scanner was used to conduct hrMCA. With the hrMCA method, we were able to screen the genomic DNA of 3 vEDS patients and one control using 3 plates (96-wells). Next, we screened for 4 glycine substitution mutations that we already knew about and 3 splicing mutations of COL3A1 using genomic DNA from patients suspected of having vEDS; we were able to identify all 7 of them. We also compared various methods of hrMCA using different instruments and fluorescence dyes. The hrMCA method allows quick and easy detection of mutations in vEDS patients, and we believe this method is appropriate for clinical use.

\section{My Experience of Clinical Training and Basic Research at Nippon Medical School}

Shenhao Wang ${ }^{1,3}$, Takayuki Aimoto ${ }^{1}$, Koichi Miyake², Nobuyuki Sakurazawa', Noriko Miyake ${ }^{2}$, Takashi Shimada ${ }^{2}$ and

Eiji Uchida ${ }^{1}$

${ }^{1}$ Department of Surgery, Nippon Medical School

${ }^{2}$ Department of Biochemistry and Molecular Biology,

Nippon Medical School

${ }^{3}$ Department of Gastroenterology,

The Second Affiliated Hospital, School of Medicine, Xi'an Jiaotong University

I have been engaged in clinical training and basic research at Nippon Medical School, participating as an assistant in endoscopic submucosal dissection (endoscopic resection for early stage gastrointestinal malignancies), and gaining valuable experience. I have also carried out basic research to evaluate the effects of systemic gene therapy using AAV-8 vectors expressing MDA-7/IL24 on pancreatic cancer. We generated an animal model of pancreatic cancer by transplanting hamster pancreatic cancer cells into the splenic lobe of the pancreas. Two weeks after transplantation, the experimental animals were divided into 2 groups as follows: a 
treatment group receiving AAV-8-MDA-7 vectors intramuscularly; and a control group receiving AAV8-EGFP vectors. Tumor growth was followed with an IVIS system, and serum IL24 concentration was measured with ELISA. All animals were monitored for survival. Injection of AAV-8-MDA-7 did not significantly inhibit tumor growth, and there was no significant increase in serum IL24 concentration in the treatment group. No significant differences were found in overall survival. These results showed that the AAV-8-MDA7 vectors used in this experiment do not express sufficient amounts of MDA-7 protein for cancer gene therapy. Our next task is to produce new AAV-8-MDA7 vectors with higher MDA-7 protein titers.

\section{Angioscopic Study of Silent Plaque Rupture in Patients with Stable Ischemic Heart Disease}

Zuoyan Wang', Shigenobu Inami', Sonoko Kirinoki ${ }^{1}$, Eisei Yamamoto ${ }^{1}$, Gen Takagi', Satoshi Aoki', Koji Kato', Hitoshi Takano', Kuniya Asai ${ }^{1}$, Masahiro Yasutake', Masamichi Takano ${ }^{2}$, Masanori Yamamoto', Takayoshi Ohba ${ }^{2}$ and Kyoichi Mizuno ${ }^{1}$

${ }^{1}$ Department of Internal Medicine, Nippon Medical School

${ }^{2}$ Department of Internal Medicine,

Nippon Medical School Chiba Hokusoh Hospital

Plaque disruption plays a key role in the development of acute coronary syndromes. However, the clinical profile of asymptomatic plaque disruption in stable ischemic heart disease has not been well evaluated. Our aim was to investigate the frequency and determinants of silent plaque disruption (SPD) in patients with stable ischemic heart disease using coronary angioscopy. Forty-one patients with stable angina or old myocardial infarction (OMI) without any complaints within 3 months were enrolled. Angioscopy of 49 nonischemic-related arteries was performed. Silent plaque SPD was found in 12 patients with stable ischemic heart disease (12/41 [29.3\%]), and the frequency of SPD in non-ischemic-related arteries was $26.5 \%(13 / 49)$. A significantly higher frequency of SPD was noted in yellow plaques than in white plaques $(35.3 \%$ vs $6.7 \%, \quad \mathrm{P}=0.043)$. Overall, the independent clinical risk factors of SPD in nonischemic-related arteries were diabetes mellitus $(\mathrm{P}=$ 0.018; OR: 18.8209; 95\% CI: 1.6525 to 214.3523 ) and hypertension ( $\mathrm{P}=0.0313$; OR: 6.6485; 95\% CI: 1.1850 to 37.3019). These results suggest silent plaque disruptions are commonly present in non-ischemicrelated coronary arteries in stable ischemic heart disease and that the determinants are diabetes mellitus and hypertension.

16. The Effects of Thymol and Thymol Glucoside in Comparison with Those of Monensin on the in vivo Metabolism of Dairy Cattle and on in vitro Rumen Fermentation

Jin-suk Jeong, Ju-hwan Lee, Hisao Itabashi and Nobuhiro Kimura

Division of Animal Nutrition,

Nippon Veterinary and Life Science University

Purpose: The object of this study was to investigate the dose-response effects of monensin (MO) and thymol (TM) on in vitro rumen fermentation and in vivo blood biochemical and hematological variables in dairy cows. We also evaluated thymol glucoside (TMG) as a possible alternative to MO.

Methods: Rumen fluids containing $\mathrm{MO}$ and TM were incubated at $39^{\circ} \mathrm{C}$ for $6 \mathrm{hr}$ and shaken at 30 rpm to create in vitro fermentation. Four dairy cows were fed a diet containing TMG synthesized from TM, and their blood parameters for biochemical and hematological variables were analyzed with a Beckman-Synchron CX5. The volatile fatty acids (VFAs) in the resultant solutions were analyzed with gas chromatography, and the degradation of TMG and TMG intermediates was measured with high performance liquid chromatography.

Results: TM and MO showed some similarities in their effects on in vitro fermentation (reduction of methane and ammonia-N levels) as well as some differences (volatile fatty acid composition and acetate/propionate $[\mathrm{A} / \mathrm{P}]$ ratio). TMG had a strong immuno-stimulatory effect, producing a maximum $56 \%$ increase in white blood cell counts, which 
suggests its potential for use in strengthening the immune system. Additionally, TMG reduced the A/ $\mathrm{P}$ ratio by $15 \%$ and methane production by $11 \%$, both of which are positive indices for fermentation efficiency. This study indicates that TMG might be better than TM for rumen fermentation, offering better results with fewer side effects.

\section{Experience of Laparoscopic Adrenalectomy in Japan}

Sherif Zeidan ${ }^{1}$, Takehito Igarashi ${ }^{1}$, Yoshiharu Nakamura', Haruki Akasu', Ritsuko Okamura ${ }^{1}$, Kayo Miyawaki ${ }^{1}$ and

Kazuo Shimizu ${ }^{1}$

${ }^{1}$ Division of Endocrine Surgery, Department of Surgery, Nippon Medical Schoo

${ }^{2}$ Division of Gastrointestinal Surgery,

Department of Surgery, Nippon Medical School

Background: Laparoscopic adrenalectomy (LA) has become the preferred approach for removal of the adrenal gland. Many published studies support the use of LA over open adrenalectomy, suggesting many advantages to laparoscopy: less postoperative pain; shorter hospital stay; and earlier return to work.

Objective: Studying LA for one year as well as acquiring the clinical skills to perform it was the purpose of my stay in Japan.

Methods: A prospective study of all patients undergoing LA for functioning and non-functioning adrenal tumors over a one-year period is in progress. The study includes preoperative presentation, operative procedure, and a short postoperative follow-up.

Results: Thirty patients who underwent laparoscopic resection of 33 adrenal tumors have been studied to date $(\mathrm{M}: \mathrm{F}$ ratio=2 : 1 ; mean age: 47.7 years). Twenty-one of the tumors were leftsided and 12 right-sided. Patient presentation, diagnosis, operative time, intraoperative blood loss, tumor sizes, postoperative pain, and postoperative hospital stay are the main points studied.

Conclusion: LA is the operation of choice in benign and carefully selected malignant adrenal tumors. It is safe and effective, but it requires adequate training as it can be technically challenging.

\section{Effects of Adipose Stem Cells on the Viability of Diced Cartilage Grafts}

\section{Hakan Orbay ${ }^{1}$, Masaaki Miyamoto², Yasuhiko Tabata ${ }^{3}$, Hiroshi Mizuno ${ }^{1}$ and Hiko Hyakusoku'}

${ }^{1}$ Department of Plastic and Reconstructive Surgery,

Nippon Medical School

${ }^{2}$ Division of Regenerative Medicine,

Nippon Medical School

${ }^{3}$ Department of Biomaterials Institute of

Frontier Medical Sciences, Kyoto University

Diced cartilage grafts are frequently used for nasal augmentation, but some resorption of cartilage is to be expected in the long term. We examined the effects of adipose stem cells (ASCs) and basic fibroblast growth factor (bFGF) on the viability of diced cartilage grafts. Thirty male Fischer rats were randomly divided into 6 groups. Diced cartilage wrapped with different materials was then grafted in each group as follows: group I: fascia; group II: Surgicel; group III: fascia + bFGF (10 $\mu \mathrm{gr} / \mathrm{graft})$; group IV: Surgicel + bFGF (10 $\mu \mathrm{gr} /$ graft); group V: fascia + ASCs; group VI: Surgicel + ASCs. ASCs were harvested from the inguinal fat pads of the rats $\left(1.08 \times 10^{6}\right.$ cells/graft). Fascia was obtained from the abdominal region, and cartilage from the ears of the rats. Weights of the grafted cartilage were recorded before implantation and after explantation. Hematoxylin and eosin, Masson's trichrome, safranin O, Van Gieson, and anti-glial fibrillary acidic protein immunohistochemical staining were done 8 weeks postoperatively. Cartilage resorption as measured by weight change was highest in groups II, IV, and VI, and the highest vessel count was in group V. The number of nucleated lacunae per unit area and the number of proteoglycan-containing areas were highest in group V. Inflammation was highest in the Surgicel groups. Elastic fibers around regenerating cartilage islands were noted on Van Gieson staining in the fascia groups but not in group II. In conclusion, ASCs significantly increase the viability of grafts, and bFGF also has a beneficial effect. 
19. In vitro Differentiation of Adipose-derived Stem Cells into Schwann Cells and in vivo Implications for Peripheral Nerve Defects

\section{Hakan Orbay, Ahmet Cagri Uysal, Kyoko Kobe, Hiroshi Mizuno and}

Hiko Hyakusoku

Department of Plastic and Reconstructive Surgery,

Nippon Medical School

Stem cell technology has evolved as a promising treatment modality for a variety of health problems that are difficult to treat. Peripheral nerve injury is one of them. This study involved both in vitro and in vivo steps. Adipose-derived stem cells (ASCs) were differentiated in vitro with a Schwann cell induction medium. Immunohistochemical staining for Schwann cell proteins (S100, Integrine $\beta 4$ and NGFR p75) and real-time PCR to detect the proteins' genes were employed. The cells were transplanted into nerve defects in the sciatic nerves of Fischer rats. The results were evaluated functionally (walking track analysis, nerve conduction velocity test) and histologically ( immunohistochemical staining, hematoxylin and eosin staining, toluidine blue staining, scanning electron microscopy). The best nerve regeneration in terms of myelinated fiber density and myelinated/unmyelinated fiber ratio was in the ASC and differentiated ASC groups. The results of the walking track analysis and nerve conduction velocity test were grossly parallel with the histologic results. Moreover, our data strongly suggested that a small portion of the injected ASCs were differentiated into Schwann cells in vivo and that differentiated ASCs sustained their Schwanncell characteristics in vivo. The results encourage further studies and indicate a promising future for the clinical application of ASCs in treating nerve defects.

20. Measuring Metabolites during Hemorrhagic Shock Using an Intraluminal Microdialysis Technique
Ran Zhu, Tomohiko Masuno, Takayuki Asakura, Shinichiro Shiraishi, Norio Sato and Hiroyuki Yokota

Department of Emergency and Critical Care Medicine,

Nippon Medical School

Introduction: Gut injury and barrier dysfunction occurring in the process of traumatic/hemorrhagic shock and resuscitation accelerate systemic inflammatory response and damage the lungs and other organs. The exact mechanism of the damage and recovery process in the gut epithelial barrier is not known. Microdialysis is a good way to explore what really happens in the interstitial spaces of many organs, including the gut. Microdialysis involves introducing a catheter with a semipermeable membrane into the interstitial space, and collecting the dialysate serially, which contains important cellular and metabolite information, through the membrane. We have investigated gut injury in a rat model of traumatic/hemorrhagic shock using this technique.

Methods: Traumatic/hemorrhagic shock was induced in rats, with mean arterial pressure maintained at $30 \mathrm{mmHg}$ for 45 mins. Two microdialysis catheters were fixed onto the mucosa and serosa of the ileum before shock, and dialysate samples were collected every 60 minutes to analyze for glycerol, glucose, lactate, pyruvate, and glutamate throughout the whole experiment period of almost 10 hours.

Results: Glycerol, glucose, lactate, and pyruvate levels in both the mucosal and serosal samples were increased in the shock group during the first hour of resuscitation; they then returned to normal. No increase was observed in the sham-shock group. Glutamate levels were increased in the later phase only in samples taken from the mucosal side.

Conclusion: We detected the changes in mucosal metabolites throughout ischemia, reperfusion, and recovery phase using a microdialysis technique. The increase in intraluminal glutamate levels in the later phase may be related to the restitution mechanism of the damaged epithelial barrier function, acting either as a direct fuel or substrate to form glutamine for the epithelial cells. 